\title{
Percepción de la calidad y satisfacción estudiantil en la escuela de administración de una universidad pública, Apurímac, 2019
}

\author{
Dr. Julio César Machaca Mamani \\ jcmachaca@unajma.edu.pe \\ https://orcid.org/0000-0003-4866-3885 \\ Universidad Nacional José María Arguedas \\ Perú, Andahuaylas \\ Dra. Rocío Cahuana Lipa \\ rcahuanal@utea.edu.pe \\ https://orcid.org/0000-0002-7671-5585 \\ Universidad Tecnológica de los Andes \\ Perú, Andahuaylas

\section{Dra. Rosario Giovanna Machaca Mamani d01323009@uancv.edu.pe https://orcid.org/0000-0002-1606-1575} \\ Universidad Andina Néstor Cáceres Velásquez \\ Perú, Juliaca
}

\section{RESUMEN}

Objetivo: Determinar la relación entre la percepción de la calidad del servicio educativo y la satisfacción estudiantil en la Escuela de Administración de Empresas, Universidad José María Arguedas, Apurímac 2019. Método: Hipotético deductivo, básica, de nivel descriptivo, con enfoque cuantitativo, tipo correlacional, bivariado, diseño no experimental y transversal. Muestra estratificado sujeto a muestreo probabilístico de 149 individuos. Instrumento validado de 44 ítemes y fiable con Alfa de Cronbach de 0,897. Resultados y conclusión: El valor de significancia no fue superior a 0,05 en ninguno caso, en consecuencia, quedaron rechazados todas las hipótesis nulas, aceptándose las alternas. A nivel de género, $55 \%$ fue femenino y $45 \%$ masculino, sin embargo, son las féminas la que demostraron menor satisfacción considerando el semestre y la edad alcanzada. Finalmente se halló nivel alto y directo de relación $0,765^{* *}$ a una cota del $95 \%$ de confianza; evidenciando que, la calidad es influenciante para obtener satisfacción. A nivel 
de dimensiones, existió relación alta y positiva entre la fiabilidad y la satisfacción con rho $0,739^{* *}$ y la capacidad de respuesta con rho $0,775^{* *}$. Además, la tangibilidad y la seguridad registraron moderada relación positiva con rho $0,569^{* *}$ y $0,486 * *$ respectivamente y relación baja en la empatía con rho $0,362 * *$.

Palabras clave: Percepción de la calidad, satisfacción y servicio educativo 


\title{
Perception of quality and student satisfaction in the management school of a public university, Apurímac, 2019
}

\begin{abstract}
Objective: To determine the relationship between the perception of the quality of the educational service and student satisfaction in the School of Business Administration, José María Arguedas University, Apurímac 2019. Method: Hypothetical deductive, basic, descriptive level, with a quantitative approach, type correlational, bivariate, nonexperimental and cross-sectional design. Stratified sample subject to probabilistic sampling of 149 individuals. Validated instrument of 44 items and reliable with Cronbach's Alpha of 0.897. Results and conclusion: The value of significance was not greater than 0.05 in any case, consequently, all the null hypotheses were rejected, accepting the alternate ones. At the gender level, 55\% were female and $45 \%$ male, however, it is the females who show the least satisfaction considering the semester and the age reached. Finally, a high and direct level of relationship $0.765^{* *}$ was found at a level of $95 \%$ confidence; evidencing that quality is influential to obtain satisfaction. At the dimension level, there was a high and positive relationship between reliability and satisfaction with rho $0.739^{* *}$ and responsiveness with rho $0.775^{* *}$. In addition, tangibility and security registered a moderate positive relationship with rho $0.569 * *$ and $0.486^{* *}$, respectively, and a low relationship in empathy with rho $0.362^{* *}$.
\end{abstract}

Keywords: Perception of quality, satisfaction and educational service

Artículo recibido: 15 enero 2022 Aceptado para publicación: 08 febrero 2022 Correspondencia: juliocesarmachacamamani400@gmail.com Conflictos de Interés: Ninguna que declarar 


\section{INTRODUCCIÓN}

Hoy en día la calidad como filosófica se ha masificado y globalizado a escala mundial y su inclusión se refleja en casi todos los asuntos de la vida humana y las instituciones en sí. Por lo tanto, la calidad aumenta la competitividad de la organización, los activos intangibles se vuelven cada vez más perfectos y previene errores en los procesos productivos. Como término referido a la prestación de servicios educativos globales, la calidad que brindan las instituciones académicas no difiere de esta realidad. (Botello, 2014).

Internacionalmente, organizaciones como la UNESCO, que promueve la ciencia, la cultura y la educación ha exteriorizado, preocupación evidente desde 1995, por los índices de la calidad educativa especialmente en países de América Latina y el Tercer Mundo a pesar de la ubicuidad de las tecnologías y los productos del conocimiento dando alerta de los primeros síntomas a nivel mundial a cerca del evidente incremento en la desatención de la calidad y de la carencia del mismo en los sistemas y procesos educativos. (Andersen, 1995).

Frente a este diagnóstico crudo pero importante, y en la misma línea de la indiferencia pedagógica de hace 20 años, la Unión Europea ha implementado en su oportunidad profundas reformas de nivel educativo basados en su autonomía dirigido exclusivamente a su educación superior, contemplada en la Declaración de Bolonia, pues gracias a esta declaración se realizó importantes reformas a nivel educativo para alinear el contenido del plan de estudios con los requisitos nacionales, mejorar la calidad y la transparencia a través del intercambio de graduados y como fuente de convergencia institucional para evitar las limitaciones fronterizas mediante la adopción de un aprendizaje cuantificado basado en créditos, y gracias a esto las universidades de hoy pueden clasificarse en orden de desempeño, como lo indican las clasificaciones QS de las universidades que utilizan la calidad de la educación como indicador. (Araya, 2017).

Por otro lado el Banco Mundial justifica la publicación del contenido de un informe publicado en 2017 y afirma que el número de nuevos ingresantes a la educación superior en América Latina y el Caribe se ha duplicado en dos décadas por lo que satisfacer las necesidades de la sociedad no salió según lo planeado de acuerdo a calidad y eficiencia, dando cuenta que el problema potencialmente se está agravando debido a que se ha incrementado la demanda educativa, y en relación inversa la oferta educativa, permanece 
estática. Configurándose en otro de los síntomas que acarreará la ausencia de la calidad. (Araya, 2017).

Asimismo, el informe indica que se debe implementar una educación superior de calidad para facilitar el desarrollo. También incorpora el principio de que la calidad debe evitar confusiones, garantizar la igualdad de oportunidades y establecer vínculos sólidos con el mercado laboral creando mejores perspectivas de empleo e ingresos dignos. La economía se basa en una regulación optimizada. El informe concluye señalando que la realidad de la educación en América Latina y el Caribe es mayor que en África en la actualidad.

A nivel nacional, muchas instituciones de educación superior han cesado sus actividades normales y no han logrado el ambiente educativo de calidad que exige la normatividad peruana vigente que establece la Superintendencia Nacional de Educación Superior Universitarias del Ministerio de Educación, en tanto, muchos otros se esfuerzan constantemente por mejorar su imagen y sostenibilidad en el mundo académico. Sabido es que el Perú destina solo el 3,7\% de su producto interno bruto (PIB) a la educación, lo que lo convierte en el país con menor inversión en América Latina en la región. Mientras tanto, países vecinos como Bolivia, Brasil y Venezuela han invertido al menos un 6\% reafirmando la sentencia económica de que no hay resultado sin inversión se refiere directamente a la calidad de la educación. (SUNEDU, 2015).

En 2018 el informe Bienal de la SUNEDU compendia la institucionalidad universitaria en el país, revelando que en la década de los noventa funcionaban 49 universidades, la mayor parte localizada en Lima y la diferencia en la costa, creadas por la otrora Ley 23733. Para ese entonces el sistema universitario carecía de operaciones y componentes de supervisión que atendiera la calidad, generándose de esta manera el inicio de las causas que dan orígenes a esta problemática nacional y que lo percibimos hoy en día.

Luego de la aparición del Consejo Nacional para la Autorización de Funcionamiento de Universidades (CONAFU) en 1995, y que por cierto hizo poco por fortalecer la calidad, se creó en el 2006 el Sistema Nacional de Evaluación, Acreditación y Certificación de la Calidad Educativa (SINEACE) por Ley 28740 con el propósito fundamental de peritar la calidad y promover el mejoramiento continuo, tanto así, que durante el 2008, el Estado emprendió medidas para variar el rumbo universitario por medio de políticas de regulación de la educación superior con clara misión supervisadora y fiscalizadora. Posteriormente durante el año 2012, se actuó radicalmente, prohibiendo la creación y los 
otorgamientos de autorizaciones para los funcionamientos de nuevas universidades y filiales por 5 años seguidos con el propósito de lograr replanteos en la política educativa por medio de una nueva legislación que permita alcanzar y garantizar la calidad educativa peruana. Finalmente, en el 2014 se aprobó la Ley 30220 ordenando a la universidad estatal y no estatal de tipo societaria y asociativa a las respectivas adecuaciones a estándares mínimos de calidad que se deben consumar para sus pertinentes promociones. Estableciéndose de esta manera nuevos dispositivos de control ante los evidentes resultados desalentadores sobre calidad educativa que se manifestaban en el país. (SUNEDU, 2015).

Consecuentemente, el Estado de manera directa se ha convertido en la entidad garantizadora de la calidad y actor principal de la política educativa y defensor de la fe pública de los usuarios, tal es así, que en su momento ha optado por las desactivaciones de la Asamblea Nacional de Rectores (ANR) y la CONAFU respectivamente otorgándole facultades plenas a la SUNEDU, con el fin superior de supervisar esencialmente la calidad universitaria. Lo que pone de manifiesto la implementación de un inevitable sistema de pronósticos que va requerir urgentemente de retroalimentación para garantizar mejores respuestas y resultados; por consiguiente se gesta como consecuencia el control de dicho pronóstico como parte integrante y fundamental de este feedback, para determinar si este pronóstico manifiesta desviaciones en sus resultados reales como consecuencia de los cambios esenciales en los procesos o en su defecto en la aleatoriedad de las circunstancias. Por último, la encuesta nacional de egresados de universidades (ENEU) del 2014 ha reflejado la realidad universitaria dando cuenta de mejoramientos en las infraestructuras de las universidades en general recibiendo calificaciones de excelencia en $60 \%$ y buenos en $40 \%$ respectivamente, mientras que los servicios prestados de manera complementaria han sido apreciados en $52 \%$ como buenos y $10 \%$ como excelentes. Por otro lado, el componente docente para las entidades particulares de tipo asociativas era apreciadas en $28 \%$ como excelentes y $57 \%$ como buenos, mientras que las de tipos societarios eran vistas por el $23 \%$ de los diligenciados como excelentes y $61 \%$ como buenos; dejando relegados a las universidades públicas con apreciaciones que solamente alcanzan el $9 \%$ de excelencia y $55 \%$ como buenos. Concluyentemente las encuestas imprimen, además, heterogeneidad en varios aspectos quedando demostrado que las entidades universitarias no cuentan con suficientes estímulos económicos para invertir en calidad advertido por 
lo menos en el ámbito público. Hechos que evidencian que los pronósticos, luego de la implementación y aplicación de la última legislación universitaria del 2014, están exteriorizando sus primeros resultados. (INEI, 2015).

A nivel regional, la Universidad José María Arguedas, ha logrado licenciarse en el 2017 por seis años consecutivos revalidándose el 2023. Cuenta al momento con seis escuelas (Ingeniería de Sistemas, Ambiental y Agroindustrial; Administración de Empresas, Educación Primaria Intercultural y Contabilidad) todas integradas en 2 facultades que desarrollan 29 líneas de investigación. En tanto, de su envergadura reducida la universidad logró ejecutar la mayor parte de su presupuesto constantemente desde el 2013 tal como lo revelan sus estados financieros en favor de sus 1606 estudiantes, 135 docentes y 122 trabajadores administrativos. (UNAJMA, 2018).

Contextualmente y de manera final se logra desprender la realidad por la cual atraviesa el objeto de estudio, advertida con una población de 388 sujetos matriculados, constituyéndose a la fecha en la opción de formación profesional con mayor demanda en Andahuaylas. En la actualidad cuenta con suficientes servicios para su funcionamiento, sin embargo, no ha accedido a ninguna certificación o acreditación de calidad evidenciando la obligación inevitable de perfeccionar estudios sobre el particular, que contribuyan a las indispensables autoevaluaciones y/o autodiagnósticos antes de lograr dichas certificaciones y acreditaciones en el espectro académico nacional e internacional.

\section{MATERIALES Y MÉTODOS}

Según Bernal (2006) se trata de un estudio bivariado, descriptivo, enfocado cuantitativamente, con diseño no experimental, transeccional y de nivel correlacional entre las variables percepción de la calidad hallado a través del arquetipo Servperf de Zeithalm y Jo Bitner (2009) y la satisfacción del estudiantado universitario hallado mediante el modelo de necesidades educativas de Gento y Vivas (2003) en clara referencia a los servicios educativos, donde se da usanza al método hipotético deductivo. (Hernández Sampieri et al, 2014).

$$
n=\frac{(Z)^{2} \cdot(p \cdot q \cdot N)}{(E)^{2} \cdot(N-1)+(Z)^{2} \cdot(p \cdot q)}
$$

Nota. $\mathrm{N}$, es la muestra inicial, $\mathrm{Z}$ es el nivel de confianza, p es la probabilidad de éxito, $\mathrm{q}$ es la probabilidad de fracaso, $\mathrm{N}$ es la población y $\mathrm{E}$ es el margen de error 


\subsection{Población y muestra}

\section{Tabla 1}

Población y muestra estratificada

\begin{tabular}{|c|c|c|c|c|c|c|c|c|c|c|c|c|}
\hline Turno & M & $\mathrm{F}$ & $\mathrm{C} 1$ & CII & CIII & CIV & $\mathrm{CV}$ & $\mathrm{CVI}$ & CVII & CVIII & CIX & CX \\
\hline Mañana & 93 & 102 & 43 & 0 & 45 & 0 & 42 & 0 & 35 & 0 & 30 & 0 \\
\hline Tarde & 100 & 93 & 0 & 45 & 0 & 43 & 0 & 39 & 0 & 34 & 0 & 32 \\
\hline Total & 193 & 195 & 43 & 45 & 45 & 43 & 42 & 39 & 35 & 34 & 30 & 388 \\
\hline Estratos & 67 & 82 & 17 & 17 & 17 & 17 & 16 & 15 & 13 & 13 & 12 & 12 \\
\hline
\end{tabular}

Nota. $M=$ masculino, $F=$ femenino, $C=$ Ciclo, extraído de registros académicos UNAJMA

En función a las necesidades de la investigación se ha visto por conveniente la utilización de la encuesta y el diligenciamiento del cuestionario a 149 estudiantes, unisex, fraccionando 22 reactivos para cada segmento del instrumento y distribuidos en las cinco dimensiones para la variable $\mathrm{X}$ con 0,826 y tres para la variable $\mathrm{Y}$ con 0,801 de confiabilidad excelente dentro del Alfa de Cronbach, respectivamente, complementado por la validación de su consistencia interna, coherencia y propósitos; lo que ha permitido otorgarle validez al instrumento de aplicación de campo. Finalmente, su pilotaje se llevó a cabo con el $10 \%$ de la estratificación.

\section{RESULTADOS Y DISCUSIÓN}

\section{De los resultados generales se dice:}

\section{Tabla 2}

Cruzada tabulación del género versus la satisfacción

\begin{tabular}{clcccccc}
\hline Género & & T.I. & P.S. & S. & B.S. & T.S. & Total \\
\hline M & Recuento & 3 & 11 & 40 & 13 & 0 & 67 \\
& \% del total & $4,5 \%$ & $16,4 \%$ & $59,7 \%$ & $19,4 \%$ & $0,0 \%$ & $45,0 \%$ \\
F & Recuento & 3 & 17 & 44 & 14 & 4 & 82 \\
& \% del total & $3,7 \%$ & $20,7 \%$ & $53,7 \%$ & $17,1 \%$ & $4,9 \%$ & $55,0 \%$ \\
\multirow{3}{*}{ Total } & Recuento & 6 & 28 & 84 & 27 & 4 & 149 \\
& \% del total & $4,0 \%$ & $18,8 \%$ & $56,4 \%$ & $18,1 \%$ & $2,7 \%$ & $100,0 \%$ \\
\hline
\end{tabular}

Nota. $M=$ Masculino, $F=$ femenino, T.I = Total insatisfacción, $P . S=$ Poca satisfacción, $S=$ Satisfecho, B.S = Bastante satisfacción, T.S = Total satisfacción .

De la tabla 2 se advierte que los varoniles ha encontrado satisfacción en 59,7\% (40 estudiantes), por su parte 19,4\% halló bastante satisfacción, 16,4\% poca satisfacción, $4,5 \%$ total insatisfacción y ninguno total satisfacción. Por otro lado, las féminas dan 
cuenta de alcanzar satisfacción en 53,7\% (44 estudiantes), así mismo, 20,7\% halló poca satisfacción, $17,1 \%$ bastante satisfacción; 3,7\% total insatisfacción y 4,9\% total satisfacción.

\section{Tabla 3}

Cruzada tabulación de la edad versus la satisfacción

\begin{tabular}{|c|c|c|c|c|c|c|c|}
\hline \multicolumn{2}{|c|}{ Edad cumplida } & \multirow{2}{*}{$\begin{array}{c}\text { T.I. } \\
0\end{array}$} & \multirow{2}{*}{$\begin{array}{c}\text { P.S. } \\
0\end{array}$} & \multirow{2}{*}{$\begin{array}{c}\text { S. } \\
1\end{array}$} & \multirow{2}{*}{$\begin{array}{c}\text { B.S. } \\
1\end{array}$} & \multirow{2}{*}{$\begin{array}{c}\text { T.S. } \\
0\end{array}$} & \multirow{2}{*}{$\begin{array}{c}\text { Total } \\
2\end{array}$} \\
\hline 16 años & Recuento & & & & & & \\
\hline & $\%$ del total & $0,0 \%$ & $0,0 \%$ & $0,7 \%$ & $0,7 \%$ & $0,0 \%$ & $1,3 \%$ \\
\hline \multirow[t]{2}{*}{17 años } & Recuento & 0 & 0 & 3 & 2 & 1 & 6 \\
\hline & $\%$ del total & $0,0 \%$ & $0,0 \%$ & $2,0 \%$ & $1,3 \%$ & $0,7 \%$ & $4,0 \%$ \\
\hline \multirow[t]{2}{*}{18 años } & Recuento & 2 & 7 & 13 & 8 & 1 & 31 \\
\hline & $\%$ del total & $1,3 \%$ & $4,7 \%$ & $8,7 \%$ & $5,4 \%$ & $0,7 \%$ & $20,8 \%$ \\
\hline \multirow[t]{2}{*}{19 años } & Recuento & 1 & 8 & 17 & 4 & 0 & 30 \\
\hline & $\%$ del total & $0,7 \%$ & $5,4 \%$ & $11,4 \%$ & $2,7 \%$ & $0,0 \%$ & $20,1 \%$ \\
\hline \multirow[t]{2}{*}{20 años } & Recuento & 1 & 5 & 17 & 4 & 0 & 27 \\
\hline & $\%$ del total & $0,7 \%$ & $3,4 \%$ & $11,4 \%$ & $2,7 \%$ & $0,0 \%$ & $18,1 \%$ \\
\hline \multirow[t]{2}{*}{21 años } & Recuento & 1 & 4 & 16 & 3 & 1 & 22 \\
\hline & $\%$ del total & $0,7 \%$ & $0.7 \%$ & $10,7 \%$ & $2,0 \%$ & $0,7 \%$ & $14,8 \%$ \\
\hline \multirow[t]{2}{*}{22 años } & Recuento & 0 & 6 & 7 & 1 & 0 & 14 \\
\hline & $\%$ del total & $0,0 \%$ & $4,0 \%$ & $4,7 \%$ & $0,7 \%$ & $0,0 \%$ & $9,4 \%$ \\
\hline \multirow[t]{2}{*}{23 años } & Recuento & 1 & 1 & 2 & 2 & 0 & 6 \\
\hline & $\%$ del total & $0,7 \%$ & $0,7 \%$ & $1,3 \%$ & $1,3 \%$ & $0,0 \%$ & $4,0 \%$ \\
\hline \multirow[t]{2}{*}{24 años } & Recuento & 0 & 0 & 5 & 1 & 1 & 7 \\
\hline & $\%$ del total & $0,0 \%$ & $0,0 \%$ & $2,7 \%$ & $0,7 \%$ & $0,7 \%$ & $4,7 \%$ \\
\hline \multirow[t]{2}{*}{25 años } & Recuento & 0 & 0 & 3 & 1 & 0 & 4 \\
\hline & $\%$ del total & $0,0 \%$ & $0,0 \%$ & $2,0 \%$ & $0,7 \%$ & $0,0 \%$ & $2,7 \%$ \\
\hline \multirow[t]{2}{*}{ Total } & Recuento & 6 & 28 & 84 & 27 & 4 & 149 \\
\hline & $\%$ del total & $4,0 \%$ & $18,8 \%$ & $56,4 \%$ & $18,1 \%$ & $2,7 \%$ & $\begin{array}{c}100,0 \\
\%\end{array}$ \\
\hline
\end{tabular}

Nota. T.I = Total insatisfacción, $P . S=$ Poca satisfacción, $S=$ Satisfecho, B.S = Bastante satisfacción, T.S = Total satisfacción.

La tabla 3 advierte que el 56,4\% de los encuestados hallaron satisfacción, dentro del segmento de edades de 19 a 21 años, así mismo, poca satisfacción el 18,8\%; bastante satisfacción el 18,1\%, total insatisfacción el $4 \%$ y total satisfacción el 2,7\%; revelando que la edad es otro factor determinante para la calificación de la calidad, es decir, mientras más edad se cumple mejor se percibe la satisfacción. 


\section{Tabla 4}

Frecuencias de la variable $X$

\begin{tabular}{lcc}
\hline Calidad & F. & \% \\
\hline Total desacuerdo & 8 & 5,4 \\
En desacuerdo & 33 & 22,1 \\
Medianamente de acuerdo & 58 & 38,9 \\
De acuerdo & 46 & 30,9 \\
Totalmente de acuerdo & 4 & 2,7 \\
Total & 149 & 100,0 \\
\hline
\end{tabular}

Nota. $F=$ frecuencia $y \%=$ porcentaje

La tabla 4 destaca mayoritariamente que el 38,9\% (58) estudiantes evidenciaron haber estado medianamente de acuerdo con la calidad percibida en la escuela de Administración.

\section{Tabla 5}

Frecuencias de la variable $Y$

\begin{tabular}{lcc}
\hline Satisfacción & F. & \% \\
\hline Total insatisfacción & 6 & 4,0 \\
Poca satisfacción & 28 & 18,8 \\
Satisfacción & 84 & 56,4 \\
Bastante satisfacción & 27 & 18,1 \\
Total satisfacción & 4 & 2,7 \\
Total & 149 & 100,0 \\
\hline
\end{tabular}

Nota. $F=$ frecuencia $y \%=$ porcentaje

La tabla 5, destaca mayoritariamente que el 56,4\% (84) estudiantes evidenciaron haberse encontrado satisfechos luego de haber percibido la calidad en la referida escuela.

\section{Tabla 6}

Frecuencias de la fiabilidad

\begin{tabular}{lcc}
\hline Dimensión 1 & F. & \% \\
\hline Total desacuerdo & 16 & 10,7 \\
En desacuerdo & 8 & 5,4 \\
Medianamente de acuerdo & 50 & 33,6 \\
De acuerdo & 56 & 37,6 \\
Totalmente de acuerdo & 19 & 12,8 \\
Total & 149 & 100,0
\end{tabular}

Nota. $F=$ frecuencia $y \%=$ porcentaje

La tabla 6, destaca mayoritariamente que el 37,6\% (56) estudiantes evidenciaron haber estado de acuerdo con la fiabilidad de la calidad ofrecida en la referida escuela. 


\section{Tabla 7}

Frecuencias de la empatía

\begin{tabular}{lcc}
\hline Dimensión 2 & F. & \% \\
\hline Total desacuerdo & 3 & 2,0 \\
En desacuerdo & 17 & 11,4 \\
Medianamente de acuerdo & 46 & 30,9 \\
De acuerdo & 52 & 34,9 \\
Totalmente de acuerdo & 31 & 20,8 \\
Total & 149 & 100,0 \\
\hline
\end{tabular}

Nota. $F=$ frecuencia $y \%=$ porcentaje

La tabla 7, destaca mayoritariamente que el 34,9\% (52) estudiantes evidenciaron haber estado de acuerdo con la empatía de la calidad ofrecida en la referida escuela.

\section{Tabla 8}

Frecuencias capacidad de respuestas

\begin{tabular}{lcc}
\hline Dimensión 3 & F & \% \\
\hline Total desacuerdo & 14 & 9,4 \\
En desacuerdo & 18 & 12,1 \\
Medianamente de acuerdo & 64 & 43,0 \\
De acuerdo & 22 & 14,8 \\
Totalmente de acuerdo & 31 & 20,8 \\
Total & 149 & 100,0 \\
\hline
\end{tabular}

Nota. $F=$ frecuencia $y \%=$ porcentaje

La tabla 8, destaca mayoritariamente que el $43 \%$ (64) estudiantes evidenciaron haber estado medianamente de acuerdo con la capacidad de respuestas fiabilidad de la calidad ofrecida en la referida escuela.

\section{Tabla 9}

Frecuencias de la tangibilidad

\begin{tabular}{lcc}
\hline Dimensión 4 & F & \% \\
\hline Total desacuerdo & 26 & 17,4 \\
En desacuerdo & 21 & 14,1 \\
Medianamente de acuerdo & 49 & 32,9 \\
De acuerdo & 49 & 32,9 \\
Totalmente de acuerdo & 4 & 2,7 \\
Total & 149 & 100,0 \\
\hline
\end{tabular}

Nota. $F=$ frecuencia $y \%=$ porcentaje 
La tabla 9, destaca mayoritariamente que el 32,9\% (49) estudiantes evidenciaron haber estado de acuerdo y medianamente de acuerdo respectivamente con la tangibilidad de la calidad ofrecida en la referida escuela.

\section{Tabla 10}

Frecuencias de la seguridad

\begin{tabular}{lcc}
\hline Dimensión 5 & F & \% \\
\hline Total desacuerdo & 13 & 8,7 \\
En desacuerdo & 15 & 10,1 \\
Medianamente de acuerdo & 69 & 46,3 \\
De acuerdo & 40 & 26,8 \\
Totalmente de acuerdo & 12 & 8,1 \\
Total & 149 & 100,0 \\
\hline
\end{tabular}

Nota. $F=$ frecuencia $y \%=$ porcentaje

La tabla 10, destaca mayoritariamente que el 46,3\% (69) estudiantes evidenciaron haber estado medianamente de acuerdo con la seguridad de la calidad ofrecida en la referida escuela.

\section{Tabla 11}

Correlación de variables y dimensiones

\begin{tabular}{lcccccc}
\hline $\begin{array}{c}\text { Variable / } \\
\text { Dimensión }\end{array}$ & $\begin{array}{c}\text { Calidad } \\
\text { de } \\
\text { servicio }\end{array}$ & Fiabilidad Empatía & $\begin{array}{c}\text { Capacidad } \\
\text { de } \\
\text { respuestas }\end{array}$ & Tangibilidad & Seguridad \\
\hline $\mathrm{N}$ & 149 & 149 & 149 & 149 & 149 & 149 \\
Significación & 0,000 & 0,000 & 0,000 & 0,000 & 0,000 & 0,000 \\
$\begin{array}{l}\text { Satisfacción } \\
\text { estudiantil }\end{array}$ & $0,765 * *$ & $0,739 * *$ & $0,362^{* *}$ & $0,775^{* *}$ & $0,569 * *$ & $0,486 * *$ \\
\hline
\end{tabular}

Nota. Rho Spearman de 2 variables y 5 dimensiones

La tabla 11 revela que para todos los casos el nivel de significación ha sido menor a 0,05 lo que ha ocasionado el rechazo de todas las hipótesis de negación y por lo tanto se han aceptado todas las alternas. Paralelamente, todos los valores resultantes de las correlaciones han sido positivos destacando moderación y alta correlación sostenidos a cotas de confianza estimadas del $95 \%$.

\section{DISCUSIÓN}

Considerando las aceptaciones de las hipótesis alternativas y general del estudio, se ha podido afirmar que los hallazgos guardan relaciones similares con otras pesquisas nacionales. 
En referencia a Arce (2018) también se logró adaptar el instrumento de Gento y Vivas (2003) para la construcción de reactivos e indicadores de la segunda parte del instrumento. De las conclusiones se dice que la relevancia y satisfacción demostraron relación moderada de 0,590. Eficiencia, eficacia y satisfacción con $r=0,491$ y Equidad y satisfacción con $r=0,539$. Muy semejante resultó el abordaje del tema central del estudio, sin embargo, se diferenció por las teorías adoptadas, es decir, por las de Municio, que no fueron tomadas en cuenta por el presente estudio.

Semejante caso sucedió con Cerna (2017) que concluyó, con la presencia de relación de las dos variables iguales al caso Andahuaylino. Además, la Calidad de servicio y satisfacción con r, alta de 0,895. Calidad académica y satisfacción con r, moderada de 0,585. Calidad de gestión y satisfacción con $\mathrm{r}$, alta de 0,789 y calidad de mantenimiento y satisfacción con r, moderada de 0,654. Para nuestro asunto en particular el estudio coincidió en gran parte de la metodología empleada, sin embargo, los resultados difieren de la óptica de estudiantes matriculados en una universidad pública y en una privada como es el caso de la Universidad César Vallejo.

De manera disímil a los dos anteriores antecedentes, Franco (2017) ha buscado demostrar divergencias entre la satisfacción y la formación profesional en la Universidad del Centro del Perú, concluyendo con la presencia de diferencias significativas entre las variables centrales, expresadas en las capacidades de enseñanza y las recepciones del aprendizaje, investigación, extensión y proyección social. De las conclusiones y resultados se dice que el $73 \%$ señalaron satisfacciones con los servicios de formación profesional. La dimensión enseñanza aprendizaje representó el 91\% de las aceptaciones para la especialidad de Ciencias Naturales y Ambientales. De 88\% para Educación Física y $45 \%$ para Psicomotricidad. Concluyendo que la carrera con mejores porcentajes de satisfacción fue Ciencias Naturales y Ambientales con 86\%. De acuerdo a dichos hallazgos se logra coincidencia única con la incorporación de la variable enseñanza y aprendizaje para la variable dos y no se consideró a la presente investigación ni a la proyección y extensión social debido a que los modelos Servperf y educativo de Gento y Vivas no hacían atractivo su incorporación, a pesar de ser estudios parecidos.

Luque (2019) guarda cercana relación por el empleo del estándar ServQual modificado, logrando coincidencias en las interpretaciones, pero, no en los valores de las correlaciones, hallando: Seguridad y tangibilidad relacionados con las expectativas 
alcanzaron 0,536 y 0,528 respectivamente; por otro lado, las seguridades con la percepción lograron 0,505. Finalmente, las más elevadas correlaciones se registraron entre: Seguridad y expectativas con 0,536 ; tangibilidad y expectativas con 0,528 . Sin embargo, para el caso Andahuaylino las más altas correlaciones se dieron entre la calidad y la satisfacción como variables generales con un valor de $0,765^{* *}$ y por su parte la capacidad de respuesta y la satisfacción con $0,775^{* *}$ resultando las demás dimensiones con niveles moderados y bajo.

En referencia a los antecedentes del extranjero, se reveló concordancias con el estudio de Ortega, et al. (2015) tras el empleo del estándar ServQual adecuado y multidimensional para Europa. Sin embargo, este último no mide las percepciones sino la diferencia entre las expectativas y las percepciones de los universitarios europeos; por otro lado, dichos sujetos vislumbran su futuro en entornos globales hecho que no ocurre aun en Apurímac. Logra concordancia, al advertir que la calidad es fuente garantizadora de satisfacción y vital estrategia de competencia entre las universidades hoy en día. En consecuencia, se precisa que no todos los individuos perciben a la calidad con las mismas perspectivas, sino remítase a los casos europeos donde se valora las edades de los universitarios, su permanencia, y género. Se adujo a su vez que la calidad debe homogenizar la diversidad educativa en Europa. Se consideró las dimensiones: Comedores $(0,27)$, tangibles electrónicos $(0,55)$, tangibles diversos $(0,6)$, empatía $(0,80)$ y una combinación entre la fiabilidad, capacidades de respuestas y aseguramiento de $(0,91)$. En lo que respecta al estudio Apurimeño los hallazgos fueron disímiles: Relación alta entre la calidad y la satisfacción en general con un valor de correlación de 0,765. Así mismo para la capacidad de respuesta en relación con la misma satisfacción 0,775 y de 0,739 para la fiabilidad. De 0,362 para empatía; de 0,569 para la tangibilidad y de 0,486 para la seguridad respectivamente.

Respecto al estudio mexicano de Surdez, et al. (2018) se ha logrado concordancia categórica al señalar que la capacidad instalada es un elemento infaltable dentro de las evaluaciones de la calidad. Por otro lado, se ha notado las diferencias entre los universitarios peruanos y los mexicanos en razón a los niveles de libertad y autonomía para elegir sobre su futuro profesional sin mayor reparo. Los hallazgos dieron cuenta de correlaciones positivas de los mejores alumnos y la satisfacción, pero no es ilógico aseverar que los sujetos sobresalientes siempre conseguirán satisfacción. Además, con 
referencia al caso apurimeño se ha logrado concordancias a cerca de las orientaciones y tutorías de los docentes, pero en menor grado de las actualizaciones de enseñanza y aprendizaje. Otro elemento no diferenciador es que a los mexicanos les atrae por encima de los conocimientos teóricos, la praxis. Finalmente, el 56\% del estudiantado mexicano advierten insatisfacción parcial y $25 \%$ insatisfacción total especialmente en el tema infraestructural, por otro lado, la autorrealización aviso satisfacción. Respecto a la creatividad y el academicismo demostraron libertad y autonomía. Por último, existió correlación 0,899 Spearman entre los universitarios sobresalientes y la autorrealización. Tumino y Poitevin (2014) aproximaron sus investigaciones en una universidad argentina utilizando nuevamente el patrón ServQual, advirtiendo que los discernimientos acerca de la calidad pueden resultar afectadas por las propias esperanzas, circunstancias y culturas; en consecuencia, los instrumentos de medición potencialmente utilizados deberían estar contextualizados a las idiosincrasias de las poblaciones, hecho congruente los análisis de la presente investigación. La pesquisa dio cuenta además que en relación a la comunicación y al clima organizacional se han manifestado diferencias significativas y del mismo modo las destrezas para la ejecución de los procesos administrativos. Se concluyó afirmando que la mejor forma de lograr aprendizajes es a través de las interacciones prácticas complementadas por las teóricas.

Alvarado, et al. (2016) analizaron los grados de percibimientos de la calidad en los universitarios y de los estudiantes de Tecnológico. El primero nacional y el segundo no estatal. Lo hallazgos reflejaron que el alumnado del Instituto consideró de mayor relevancia las disposiciones de modernas infraestructuras e implementaciones tecnológicas a diferencia de los universitarios quienes observaron en mayores proporciones de importancia los niveles académicos de los catedráticos. Así mismo se observó, que el $79 \%$ de estudiantes de tecnológico efectúan paralelamente actividades complementarias a su formación profesional, es decir, trabajan y estudian a la par; mientras que los universitarios hacen lo mismo, pero en $29 \%$. Es preciso indicar que para el caso peruano no se ha considerado el indicador dedicación laboral y fuente laboral como actividades paralelas a la formación superior, lo que resulta siendo diferente al estudio mexicano. Concluyendo que las percepciones a cerca de la calidad son distintas para ambos tipos de estudiantes. 
En el epílogo el estudio guatemalteco de Erazo, et al. (2017) llevó a efecto comparaciones de los grados de satisfacción en diversas localidades, como el metropolitano y regional de Guatemala, hecho que fue una limitación en el presente estudio peruano. Los hallazgos confirmaron presencia de satisfacción educativa en los niveles de formación pertinente categorizada por satisfactoriamente mediana a diferencia del caso peruano que fue calificada mayoritariamente por satisfactorio, en consecuencia, se puede sostener que las latitudes donde están ubicados las sucursales unidades de enlace y/o filiales, influyen en los discernimientos acerca de la calidad y por lo tanto de la satisfacción.

\section{CONCLUSIONES}

1) Presencia de nivel de relación alto de $0,765^{* *}$ Spearman a una cota del $95 \%$ de confianza entre la percepción de la calidad del servicio educativo y la satisfacción en la Escuela de Administración de la UNAJMA, sosteniendo que dicha calidad es influenciante para lograr la satisfacción, es decir, que cuanto mejor sea evaluada la calidad, mayor será la satisfacción.

2) Presencia de relación alto de $0,739 * *$ Spearman a una cota del $95 \%$ de confianza entre la fiabilidad de la calidad del servicio educativo y la satisfacción, afirmando que dicho componente es influenciante para hallar satisfacción, es decir, que cuanto mejor sea evaluada la fiabilidad mayor será la satisfacción.

3) Presencia de relación bajo de $0,362 * *$ Spearman a una cota del $95 \%$ de confianza entre la empatía de la calidad del servicio educativo y la satisfacción, afirmando que dicho componente es influenciante para hallar satisfacción, es decir, que cuanto mejor sea evaluada la empatía mayor será la satisfacción.

4) Presencia de relación alto de $0,775^{* *}$ Spearman a una cota del $95 \%$ de confianza entre la capacidad de respuestas de la calidad del servicio educativo y la satisfacción, afirmando que dicho componente es influenciante para hallar satisfacción, es decir, que cuanto mejor sea evaluada dicha capacidad para dar respuesta mayor será la satisfacción.

5) Presencia de relación moderado de 0,569** Spearman a una cota del $95 \%$ de confianza entre la tangibilidad de la calidad del servicio educativo y la satisfacción, afirmando que dicho componente es influenciante para hallar satisfacción, es decir, que cuanto mejor sea evaluada dicha tangibilidad mayor será la satisfacción. 
6) Presencia de relación moderado de $0,486 * *$ Spearman a una cota del $95 \%$ de confianza entre la seguridad de la calidad del servicio educativo y la satisfacción, afirmando que dicho componente es influenciante para hallar satisfacción, es decir, que cuanto mejor sea evaluada dicha seguridad mayor será la satisfacción.

\section{CONSIDERACIONES FINALES}

\section{Conflictos de interés}

Lo autores no declaran haber tenido ningún conflicto de intereses.

\section{LISTA DE REFERENCIAS}

Andersen, A. (1995). Factores Humanos de la Calidad. Madrid, España: Cinco Días. Araya, L. (2017). Calidad de Servicio en Educación Superior a Distancia. España.

Alvarado, E. Morales, D. y Aguayo, E. (2016). Percepción of the quality of education; And applied case to students of the Universidad Autónoma de Nuevo León and Instituto Tecnológico de Estudios Superiores de Monterrey. Rev. La Edu. Super [Internet]. 2016;45(180):55-74. Available from: http://www.scielo.org.mx/pdf/resu/v45n180/0185-2760-resu-45-180-00055.pdf

Arce, A. (2018). Relación entre la calidad de servicios académicos y la satisfacción en los estudiantes de la Universidad Peruana Unión filial Tarapoto, 2017. [Tesis] Universidad Peruana Unión.

Bernal, C. (2006). Metodología de investigación. Segunda. México. Editor Pearson P hall. Botello JA, Chaparro Salinas E, Reyes Pérez DE. (2014). Estudio de la satisfacción de los estudiantes con los servicios educativos por Instituciones de educación del Valle de Toluca. REICE Rev. Iberoam sobre calidad, Eficiencia y cambio en Educ. [Internet]. 2014;13(2):5-26. Available from: www.rinace.net/reice/

Cerna, R. (2017). Calidad de servicio y satisfacción de los estudiantes de Administración en Turismo y Hotelería de la universidad César Vallejo, Sede Lima Este - 2017 [Internet]. Universidad César Vallejo; 2017. Available from: http://repositorio.ucv.edu.pe/bitstream/habdle/20.500.12692/9951/Cerna_CRL.p edf? sequence $=1 \&$ isAllowed $=y$

Erazo, E., Samayoa, C., López, C. y Rodas, G. (2017). Satisfacción Educativa en los estudiantes de la Facultad de Ciencias Médicas. [Tesis] Universidad San Carlos. Guatemala. 
Franco, Y. (2017). Satisfacción de estudiantes frente al servicio de formación profesional en la Facultad de Educación - UNCP [Internet]. Universidad Nacional del Centro; $2017 . \quad$ Available from: http://repositorio.uncp.edu/bitstream/handle/UNCP/3450/FrancoCuicapusa.pedf? $\underline{\text { sequence }=1 \& \text { isAllowed }=y}$

Gento Palacios S, Vivas García M (2003). EL SEUE: Un instrumento para conocer la satisfacción de los estudiantes universitario con su educación. Acción Pedagógica [Internet]. 2003;12(2):16-27. Available from: http://revencyt.ula.ve/storage/repo/ArchivoDocumento/accion/v12n2/art2.pef

Hernández Sampieri R, Fernández Collado C, Baptista Lucio M (2014). Metodología de la investigación. Quinta ed. México: McGraw-Hill/ Interamericana Editores S.A.

INEI (2015). Encuesta nacional de egresados universitarios y universidades, 2014 [Internet]. INEI. Lima; 2015. Available from:

https://www.inei.gob.pe/media/MenuRecursivo/publicaciones_digitales/Est/Lib1 298/Libro.pdf

Luque, M. (2019). Calidad de servicio y satisfacción en estudiantes de administración de negocios de una universidad privada de Arequipa, 2019 [Internet]. Universidad Católica San Pablo; 2019. Available from:

http://repositorio.ucsp.edu.pe/bitstream/UCSP/16119/1/LUQUE_BENAVIDES MAU_CAL.pdf

Ortega, J. Rodríguez, M. Muñoz, P. (2015). Calidad y satisfacción de los estudiantes europeos de educación superior. Construcción de una escala de servicio presencial y electrónico. [Internet]. Universidad de Salamanca; 2015. Available from:

http://10.0.80.31/pyr2019.v7nSPE.347\%0Ahttps://bibliotecavirtual.unad.edu.con /login?url=tttps://search.ebscohost.com/login.aspx?direct=true $\& \mathrm{db}=\mathrm{a} 9 \mathrm{~h} \& \mathrm{AN}=1$ $\underline{44682734 \& \text { lang }=\text { es \&site }=\text { ehost- }}$ live\%0Ahttp://dx.doi.org/10.1016/j.resu.2016.10.002\%0Ahttp://jstor.org/st

SUNEDU (2015). El modelo de licenciamiento y su implementación en el sistema universitario peruano. 24/11/2015. [Internet]. 2015;37. Available from: 
http://repositorio.minedu.gob.pe/bitstream/handle/123456789/4585/4585/El modelo de licenciamiento y su implementación en el sistema universitario peruano.pdf? sequence $=3 \&$ is Allwed $=\mathrm{y}$

Surdez, E., Sandoval, M. y Lamoyi, C. (2018). Satisfacción estudiantil en la valoración de la calidad educativa universitaria. [Tesis]. Universidad Juárez Autónoma de Tabasco. México.

Tumino, M. y Poitevin E. (2014). Evaluación de la calidad de servicio universitario desde la percepción de estudiantes y docentes: Caso de estudio. REICE Rev. Electrónica Iberoam sobre Calidad, Eficiencia y Cambio en Edu. [Internet]. 2014;12(2):6384. Available from:

http://revistas.uam.es/reice/article/view/2856/3073

UNAJMA (2018). PEI_2019_2021 APURIMAC.pdf [Internet]. 2018. P. 28. Available from:

http://www.unajma.edu.pe/transparencia/planeamiento_y_organización/PEI_201 9_2021.pedf

Zeithalm y Jo Bitner (2009). Metodología Servperf. Editorial Díaz de Santos. 